\title{
Transjugular Intrahepatic Portosystemic Shunt for the Treatment of Portal Hypertension- Induced Refractory Ascites Due to Metastatic Carcinomatous Liver Disease
}

\author{
Barbara Geeroms, MD, Gert De Hertogh, MD, PhD, \\ Ragna Vanslembrouck, MD, Hans Wildiers, MD, PhD, \\ Frederik Nevens, MD, PhD, and Geert Maleux, MD, PhD
}

\begin{abstract}
Three patients with a medical history of breast carcinoma and metastatic carcinomatous liver disease associated with severe portal hypertension and refractory ascites are presented. Transjugular intrahepatic portosystemic shunt creation was considered as a palliative treatment option and a valuable alternative to regular paracenteses in these patients. In 2 of the 3 patients, the refractory ascites was controlled for several months without need for paracentesis, and subsequently transjugular intrahepatic portosystemic shunt may provide valuable palliation and ascites control in patients with refractory ascites due to breast cancer-induced pseudocirrhosis.
\end{abstract}

\section{ABBREVIATIONS}

ALP $=$ alkaline phosphatase, ALT = alanine transaminase, AST = aspartate transaminase, e-PTFE = expanded polytetrafluoroethylene, $\gamma \mathrm{GT}=\gamma$-glutamyltransferase, INR = international normalized ratio, TIPS $=$ transjugular intrahepatic portosystemic shunt

Liver metastases from breast cancer can occasionally be associated with hepatic parenchymal changes mimicking cirrhosis, called "pseudocirrhosis" (1); in addition, this condition can result in symptoms of portal hypertension, including variceal bleeding and refractory ascites or hydrothorax (2). Definitive diagnosis of pseudocirrhosis is based on laboratory, imaging, and pathologic findings, and standard treatment consists in supportive care and regular paracentesis (2-4). The present report presents transjugular intrahepatic portosystemic shunt (TIPS) as an alternate palliative treatment option for patients with malignant pseudocirrhosis associated with severe portal hypertensionrelated symptoms. The Institutional Review Board at the authors' institution did not require approval for this type of

From the Department of Radiology (B.G., R.V., G.M.), Department of Pathology (G.D.H.), Department of General Medical Oncology (H.W.), and Department of Gastroenterology and Hepatology (F.N.), University Hospitals Leuven, Herestraat 49, B-3000 Leuven, Belgium. Address correspondence to G.M.; E-mail: geert.maleux@uzleuven.be

None of the authors have identified a conflict of interest.

() SIR, 2018

J Vasc Interv Radiol 2018; -1:4

https://doi.org/10.1016/j.jvir.2018.08.007 research. Informed consents were obtained before the TIPS procedures.

\section{PATIENT 1}

A 56-year-old woman with a medical history of a moderately differentiated ductal adenocarcinoma of the left breast was initially treated with local excision and external beam radiation therapy and 4 years later presented with pleuropulmonary and skeletal metastases. She successfully underwent different chemotherapy treatments, but 15 years after the initial diagnosis, she presented with multiple liver metastases, which were managed with combination chemotherapy based on carboplatin and gemcitabine. Although an initial response was demonstrated, follow-up computerized tomography (CT) revealed growth of the liver metastases, associated with imaging signs of portal hypertension, including splenomegaly, esophageal varices, and a small amount of ascites. Chemotherapy was switched to capecitabine, resulting in shrinkage of the liver metastases, but an increase in signs of portal hypertension, especially ascites and pleural effusion. Clinically, the ascites became refractory to medical therapy, and paracentesis twice a week was needed, which had already continued for more than 23 weeks. Malignancy could not be identified on 


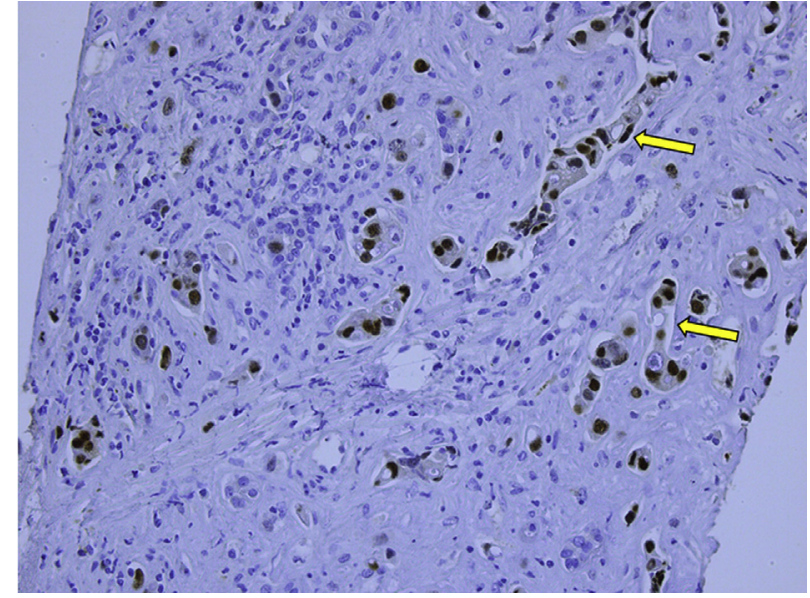

Figure 1. Transjugular liver biopsy showing dense fibrotic tissue with portal structures and some cells with nuclear atypia. Some of these cancer cells also contain mucous deposits compatible with nondifferentiated cancer cells (arrows). The tumoral proliferation is CK7 positive and CK20 negative.

repeated cytologic analysis of the ascitic fluid. A transjugular liver biopsy was performed, revealing dense fibrous tissue, portal fields containing normal hepatocytes, and nests of poorly differentiated estrogen- and progesterone-positive receptors, consistent with metastatic breast cancer cells (Fig 1). Laboratory analyses showed total bilirubin $0.99 \mathrm{mg} /$ $\mathrm{dL}$ (normal $\leq 1.0 \mathrm{mg} / \mathrm{dL}), \gamma$-glutamyltransferase $(\gamma \mathrm{GT}) 376$ $\mathrm{U} / \mathrm{L}$ (normal $\leq 50 \mathrm{U} / \mathrm{L}$ ), alkaline phosphatase (ALP) 703 $\mathrm{U} / \mathrm{L}$ (normal $\leq 240 \mathrm{U} / \mathrm{L}$ ), aspartate transaminase (AST) $83 \mathrm{U} / \mathrm{L}$ (normal $\leq 32 \mathrm{U} / \mathrm{L}$ ), alanine transaminase (ALT) $20 \mathrm{U} / \mathrm{L}$ (normal $\leq 31 \mathrm{U} / \mathrm{L}$ ), international normalized ratio (INR) $1.3(0.9-1.2)$, and platelets $72 \times 10^{9} / \mathrm{L}$ (normal $\left.150-450 \times 10^{9} / \mathrm{L}\right)$. The portosystemic pressure gradient was $16 \mathrm{~mm} \mathrm{Hg}$.

A TIPS was created to manage the refractory ascites. After placement of an expanded polytetrafluoroethylene (e-PTFE)-covered stent (Viatorr; W.L. Gore and Associates, Flagstaff, Arizona) with a nominal diameter of $10 \mathrm{~mm}$ and after dilation up to $8 \mathrm{~mm}$ in diameter, the portosystemic pressure gradient dropped to $3 \mathrm{~mm} \mathrm{Hg}$. Clinical and radiologic follow-up showed complete regression of the ascites and pleural effusion without need for repeated hospital visits. There was no clinical evidence of hepatic encephalopathy during follow-up. Three months later, the patient presented with recurrent pleural effusion without ascites. Angiographic revision of the portosystemic shunt revealed a fully patent stent graft without stenosis and a portosystemic pressure gradient of $5 \mathrm{~mm} \mathrm{Hg}$. Analysis of pleural effusion revealed malignant cells, consistent with a malignant pleural effusion. The patient died 2 months later owing to respiratory insufficiency.

\section{PATIENT 2}

A 70-year-old woman underwent surgery, radiation therapy, and adjuvant combination chemotherapy (5-fluorouracil,

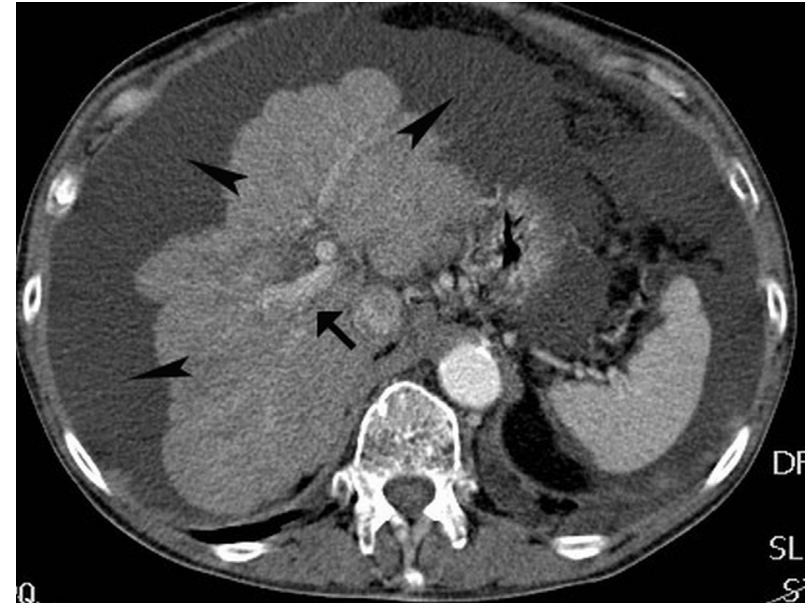

Figure 2. Axial contrast-enhanced computerized tomography reveals massive, perihepatic ascites (arrowheads). Note also the macronodular contours of the liver and the patent main portal vein (arrow).

epirubicin, and cyclophosphamide) for an invasive ductal adenocarcinoma of the right breast. Five years later, she presented with skeletal and liver metastases, which were treated with vinorelbine. However, progression of liver metastases was identified on CT, and the patient was referred to the interventional radiology department for intraarterial lobar infusion of $12 \mathrm{mg}$ mitomycin $\mathrm{C}$ per session. Follow-up CT scans revealed a response in the liver metastases, but significant shrinkage and diffuse nodularity of the whole liver associated with perihepatic ascites had developed. Several cytologic analyses of the ascitic fluid were unable to clearly show malignancy. The pseudocirrhotic signs on follow-up CTs increased, but the liver metastases remained stable (Fig 2). Clinically, the ascites became refractory to medical treatment, and weekly paracenteses were needed for 5 weeks. Laboratory analysis showed total bilirubin $1.01 \mathrm{mg} / \mathrm{dL}, \gamma \mathrm{GT} 13 \mathrm{U} / \mathrm{L}$, ALP $343 \mathrm{U} / \mathrm{L}$, AST $57 \mathrm{U} / \mathrm{L}$, ALT $55 \mathrm{U} / \mathrm{L}$, INR 0.9, and platelets $141 \times 10^{9} / \mathrm{L}$. A transjugular liver biopsy was performed, revealing nodular regenerative hyperplasia and sinusoidal dilatation, probably related to tumoral invasion of adjacent small hepatic veins (Fig 3); no fibrosis was demonstrated.

A TIPS was created with the use of an e-PTFE-covered stent (Viatorr) with a nominal diameter of $10 \mathrm{~mm}$ and after dilatation up to $8 \mathrm{~mm}$ in diameter (Fig 4); the portosystemic pressure gradient dropped from $9 \mathrm{~mm} \mathrm{Hg}$ to $2 \mathrm{~mm} \mathrm{Hg}$, and clinically the amount of ascites decreased significantly without further clinical need for paracentesis or repeated hospital visits. There were no clinical signs of hepatic encephalopathy during follow-up. Repeated ultrasonographic examination during follow-up revealed a persistent small amount of ascites in the pelvis, but without clinical need for paracenteses. A CT scan 3 months later showed growth of peritoneal metastases and a pleural effusion, but no ascites (Fig 5). Another 2 months later, the patient developed cytology-proven malignant ascites and pleural 


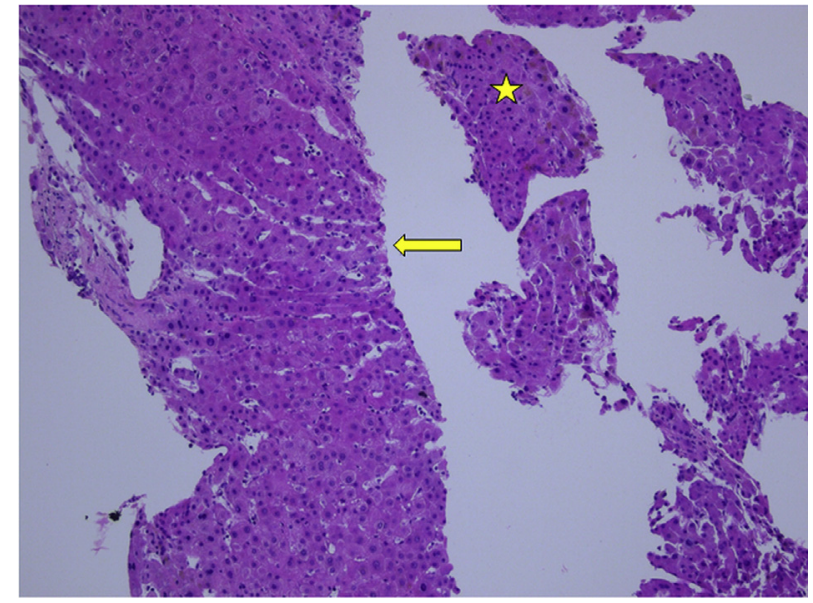

Figure 3. Liver biopsy with centrilobular sinusoidal dilatation (arrow) and mild hepatocellular cholestasis (star). These findings are nonspecific, but may be seen in the vicinity of a metastatic Iesion. Hematoxylin and eosin, $\times 100$.

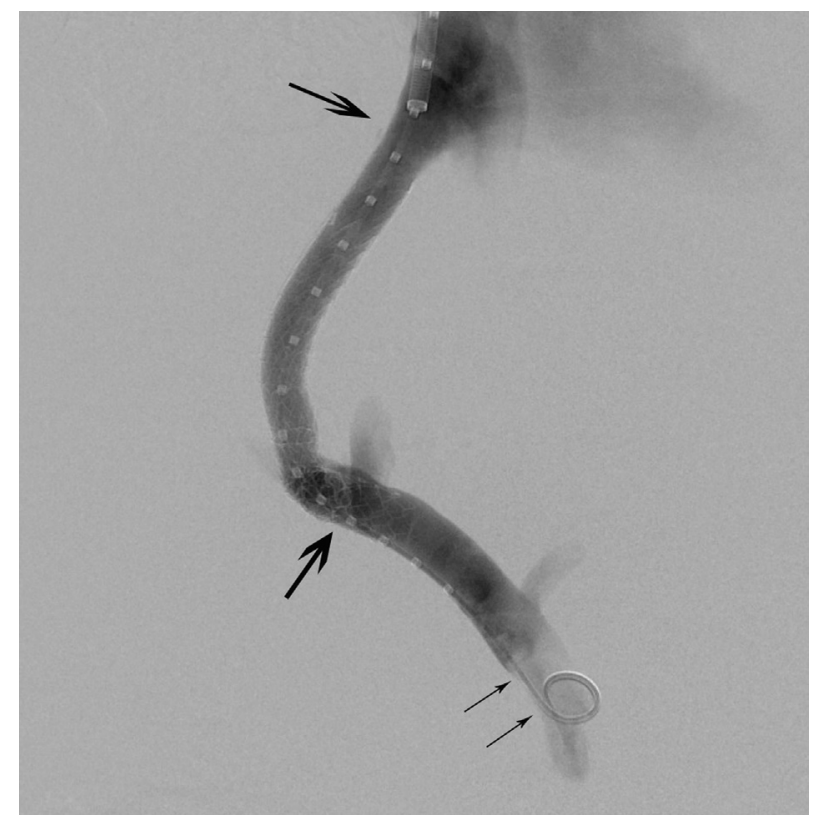

Figure 4. Contrast injection through a pigtail catheter (thin arrows) in the main portal vein after the TIPS procedure reveals a fully patent e-PTFE-covered stent (thicker arrows) from the portal vein up to the inferior vena cava.

effusion. Ultrasonography of the liver showed a patent TIPS stent. The patient died 1 month later.

\section{PATIENT 3}

A 79-year-old woman presented with omental and skeletal metastases related to a moderately differentiated lobular adenocarcinoma of the right breast. She was treated with tamoxifen and letrozole, but liver metastases developed; repeated CT imaging showed growth of the liver metastases and development of ascites. The overall liver volume and liver contours remained unchanged without nodular contour

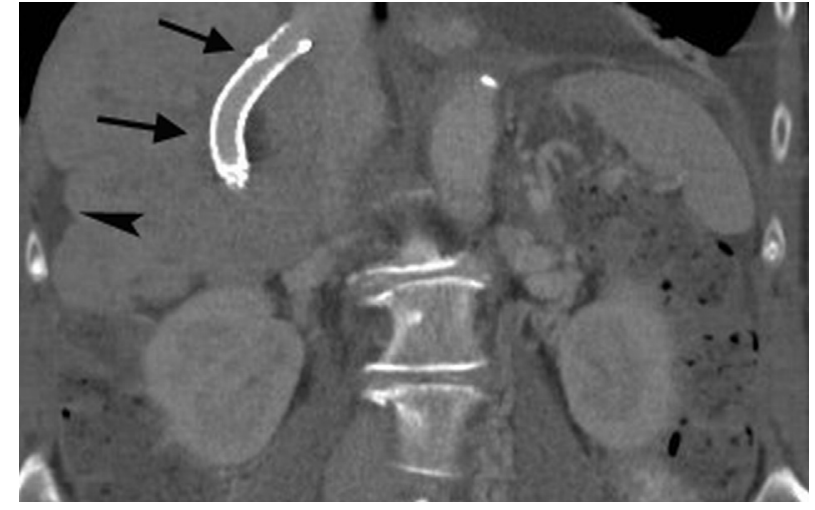

Figure 5. Reconstructed coronal computerized tomographic image demonstrates a fully patent TIPS stent (arrows). Minimal amount of perihepatic ascites (arrowhead) is seen.

irregularities; chemotherapy was switched to adriamycin because of the development of ascites. Repeated cytologic analysis did not reveal malignant cells in the ascitic fluid. Laboratory analysis showed total bilirubin $0.57 \mathrm{mg} / \mathrm{dL}, \gamma \mathrm{GT}$ $252 \mathrm{U} / \mathrm{L}$, ALP $533 \mathrm{U} / \mathrm{L}$, AST $79 \mathrm{U} / \mathrm{L}$, ALT $31 \mathrm{U} / \mathrm{L}$, INR 1.1, and platelets $250 \times 10^{9} / \mathrm{L}$. A transjugular liver biopsy showed malignant tumor nests in the portal fields with invasion of the sinusoids but without adjacent fibrosis. Hepatic pressure measurements showed a portosystemic pressure gradient of $17 \mathrm{~mm} \mathrm{Hg}$.

A TIPS was created with the use of a dedicated e-PTFEcovered stent (Viatorr) with a nominal diameter of $10 \mathrm{~mm}$ and after dilation up to $8 \mathrm{~mm}$ in diameter to treat the refractory ascites. Although the procedure was uneventful, the patient died 5 days later owing to an unknown cause. Patient recovery was uneventful in the 2 days following TIPS creation, after which she was discharged to the local care institution. Revision of the medical and radiologic records of the patient could not reveal any clinical, laboratory, or radiologic signs of liver failure, liver infarction, heart failure, intraperitoneal or intrahepatic bleeding, or sepsis.

\section{DISCUSSION}

Radiologic changes resembling cirrhosis may occur in the liver parenchyma of extensively pretreated patients with liver metastases from breast carcinoma. Imaging features including multifocal retraction of the liver capsule, enlargement of the caudate lobe, fine and diffuse nodularity, bland ascites, and portosystemic venous collaterals are defined as "pseudocirrhosis" $(1,2,5)$ and were seen in patients 1 and 2. Most of such patients present with portal hypertension, which causes many symptoms, particularly refractory ascites, as was illustrated in all 3 presented patients. Patient 1 also presented with gastroesophageal varices on gastroscopy. Different histopathologic mechanisms of pseudocirrhosis are proposed: fibrosis induced by surrounding nests of tumour cells, as in patient 1 , or nodular regenerative hyperplasia in response to chemotherapy, as in patient $2(3,6,7)$. However, owing to the presence of 
metastatic sinusoidal ingrowth and spread of tumoral nests into the portal venous system, pseudocirrhosis in patient 3 might also be related to tumoral deposits in the hepatic and portal venules, which might have the same pathophysiologic effect as thrombotic deposits, as can be seen in hepatic veno-occlusive disease.

The symptomatology of refractory ascites in the 3 presented patients was related to the pseudocirrhosis and portal hypertension rather than to malignant infiltration of the ascites, because repeated cytologic analyses remained negative for malignancy.

This report highlights the potential role of TIPS creation, with the use of dedicated e-PTFE-covered stent grafts (8), as a palliative treatment for patients with refractory ascites due to cancer-related pseudocirrhosis-induced portal hypertension. Indeed, in the first 2 patients, the refractory ascites disappeared for several months until the ascites became malignant; therefore, TIPS might be a valuable alternative to repeated paracentesis, placement of an indwelling tunneled peritoneal catheter for regular ascitic fluid drainage (9), or creation of a Denver shunt (10,11). Finally, TIPS may have the potential to keep the patient out of hospital and might improve patients' quality of life, although no dedicated analysis was performed regarding this topic.

In conclusion, in this report TIPS is presented as a potentially valuable palliative treatment option for patients presenting with refractory ascites related to breast cancerinduced pseudocirrhosis.

\section{REFERENCES}

1. Jha P, Poder L, Wang Z, Westphalen A, Yeh B, Coakley F. Radiologic mimics of cirrhosis. Am J Roentgenol 2010; 194:993-999.

2. Jüngst $C$, Krämer J, Schneider G, Lammert F, Zimmer V. Subacute liver failure by pseudocirrhotic metastatic breast cancer infiltration. Ann Hepatol 2013; 12:834-836.

3. Sas D, Clark K, Grzybicki D, Rabinovitz M, Shaw-Stiffel T. Diffuse desmoplastic metastatic breast cancer simulating cirrhosis with severe portal hypertension: a case of "pseudocirrhosis." Dig Dis Sci 2007; 42: 749-752.

4. Adike A, Karlin N, Menias C, Carey E. Pseudocirrhosis: a case series and literature review. Case Rep Gastroenterol 2016; 10:381-391.

5. Young S, Paulson E, Washington K, Gulliver D, Vredenburgh J, Baker M. CT of the liver in patients with metastatic breast carcinoma treated by chemotherapy: findings simulating cirrhosis. Am J Roentgenol 1994; 163 : 1385-1388.

6. Lee S, Chang E, Na S, Kim J, An H, Ko Y, Won H. Pseudocirrhosis of breast cancer metastases to the liver treated by chemotherapy. Cancer Res Treat 2014; 46:98-103.

7. Schouten J, Nevens F, Hansen B, et al. Idiopathic noncirrhotic portal hypertension is associated with poor survival: results of a long-term cohort study. Aliment Pharmacol Ther 2012; 35:1424-1433.

8. Geeroms B, Laleman W, Laenen A, et al. Expanded polytetrafluoroethylenecovered stent-grafts for transjugular intrahepatic portosystemic shunts in cirrhotic patients: Iong-term patency and clinical outcome results. Eur Radio 2017; 27:1795-1803.

9. Maleux G, Indesteege I, Laenen A, Verslype C, Vergote I, Prenen H. Tenckhoff tunneled peritoneal catheter placement in the palliative treatment of malignant ascites: technical results and overall clinical outcome. Radiol Oncol 2016; 50:197-203.

10. Yarmohammadi H, Brody L, Covey A, et al. Therapeutic application of percutaneous peritoneovenous (Denver) shunt in treating chylous ascites in cancer patients. J Vasc Intervent Radiol 2016; 27:665-673.

11. Lungren M, Kim C, Stewart J, Smith T, Miller M. Tunneled peritoneal drainage catheter placement for refractory ascites: single-center experience in 188 patients. J Vasc Intervent Radiol 2013; 24:1303-1308. 